\title{
Central lymph node metastasis is predictive of survival in advanced gastric cancer patients treated with D2 lymphadenectomy
}

\author{
Huiwen Lu', Bochao Zhao ${ }^{1}$, Rui Huang ${ }^{2}$, Yimeng Sun ${ }^{1}$, Zirui Zhu' ${ }^{1}$, Huimian Xu' and Baojun Huang ${ }^{1 *}$ (D)
}

\begin{abstract}
Background: The number of positive lymph nodes, which was defined as "N stage", is mostly used to predict the survival of D2-resected gastric cancer patients, not the location. A "central lymph node" (CnLN) was defined by Ikoma et al., included common hepatic, celiac and proximal splenic artery LNs. CnLNs located in the extraperigastric area are included in the D2 LN station for gastric cancer. We speculate that CnLNs can be regarded as a predictor of survival.

Methods: Eligible advanced gastric cancer patients who underwent curative resection and D2 lymph node dissection between 2004 and 2012 at our institution were identified. The frequency of CnLN metastases and risk factors affecting DFS were examined. Survival differences were assessed by log-rank tests and Kaplan-Meier curves.

Results: The study identified 1178 patients who underwent curative surgery or D2 or more extensive lymphadenectomy. A total of 342 patients had been proven to have CnLN metastasis. Larger tumor size $(P<0.001)$, more frequent lymphatic vessel invasion $(P<0.001)$, signet ring cell histology $(P=0.014)$, and more advanced pathological T stage $(P=0.013)$ were significantly related to $C n L N s$ metastasis. The patients with $C n L N$ metastasis had a poor prognosis (HR for DFS of $1.366,95 \% \mathrm{Cl}=1.138-1.640, P=0.001)$. For the $\mathrm{pN} 2 / 3$ patients, $\mathrm{CnLN}$ metastasis was associated with shorter 5-year DFS (for pN2 patients: $25.9 \%$ vs 39.3\%, $P=0.017$; for pN3 patients: $11.5 \%$ vs $23.4 \%, P=0.005$ ).
\end{abstract}

Conclusion: Gastric cancer patients with CnLN metastasis who underwent D2 resection had a poor prognosis. With the same N stage, the patients with positive CnLNs had shorter survival. CnLNs metastasis could be a supplement to $\mathrm{N}$ stage and a predictor of survival in gastric cancer patients. Large sample, multicenter, randomized clinical trials are still needed in the future.

Keywords: Gastric cancer, Lymph node metastasis, D2 lymphadenectomy, Survival

\section{Background}

Despite a decline trend in its overall incidence, gastric cancer remains the second leading cause of cancerrelated death worldwide according to the Global Cancer Statistics 2018 [1]. As a clinical doctor, our evaluation of survival outcome for advanced gastric cancer patients

\footnotetext{
*Correspondence: luhuiwen1995@163.com

1 Department of Surgical Oncology, First Affiliated Hospital of China

Medical University, No. 155 Nanjing North Street, Heping District,

Shenyang 110001, People's Republic of China

Full list of author information is available at the end of the article
}

who were treated with curative surgery and lymphadenectomy is always dependent on accurate pathological tumor staging. TNM staging, which was originally published in 1966 and has undergone several revisions, is a widely applicable classification used to guide clinical practice [2]. The definitions of " $T$ " and "M" stage have remained almost consistent, but the "N" stage has experienced a change from the "location" to the "number" of positive lymph nodes (LNs). Before the 4th edition of the American Joint Committee on Cancer (AJCC) classification system and the 2nd edition of the Japanese 
classification system, the location of positive LNs was used to define the "N" stage, which was different from that defined by the Union International against Cancer (UICC) [3, 4]. Nevertheless, some studies have suggested that the number of positive LNs reflects the tumor burden more accurately $[5,6]$. To build a homogeneous, reproducible and accurate staging system, the UICC and AJCC reached complete agreement in their definitions of TNM and stage groupings. Although the N" stage is currently defined according to the number of positive nodes, the impact of the location of positive nodes on survival outcome in gastric cancer patients is notable, as reported by several Japanese trials $[7,8]$.

Gastric cancer patients with lymph node metastasis have a poor prognosis. The knowledge gap between Western and Eastern countries has made lymph node dissection debatable. Curative resection and D2 lymph node dissection for advanced gastric cancer patients is recommended, especially in Eastern countries [9]. To the best of our knowledge, a large number of positive LNs represents a high pathological $\mathrm{N}$ stage and a poor prognosis, but the impact of the locations of positive LNs on survival outcome is unclear. Recently, Ikoma et al. has revealed that common hepatic (station no. 8), celiac (station no. 9) and proximal splenic artery (station no. 11p) LN metastasis was a reliable predictor for survival outcome in gastric cancer patients who treated with neoadjuvant chemotherapy, and the results demonstrated that the pN2 and pN3 patients with positive CnLNs experienced shorter survival than those without CnLN involvement $[8,10]$. However, no report has demonstrated the impact among GC patients who underwent D2 lymphadenectomy, without neoadjuvant chemotherapy. Whether the postoperative evaluation of the status of LN metastasis should not only count the number of positive LNs but also consider their location is worthy of consideration. The purpose of our study was to determine how anatomical location of the positive LNs influence the survival outcome of gastric cancer patients who have undergone curative surgery and D2 lymphadenectomy.

\section{Methods}

\section{Study population}

We retrospectively identified gastric cancer patients treated with curative surgery and D2 lymphadenectomy at the Department of Surgical Oncology, the First Affiliated Hospital of China Medical University between May 2004 and May 2012. The criteria for eligible patients were as follows: (1) all patients were histologically proven to be primary advanced gastric cancer via hematoxylin-eosin staining after operation; (2) the curative surgery and D2 or more extensive lymphadenectomy were performed. The patients who underwent palliative gastrectomy or with clinical and radiological evidence of peritoneal dissemination or distant metastasis were excluded from this study. (3) The patients with the history of other malignant tumors or with neoadjuvant chemotherapy or who were lost to follow-up or died within 1 month after surgery were excluded. According to the eligibility criterion above mentioned, a total of gastric cancer 1345 patients were included in this study. This study was approved by the Institutional Review Board of the Ethics Committee of China Medical University.

The study was approved by the Clinical Research Ethics Committee of the First Affiliated Hospital of China Medical University (approval No. 2016-114).

\section{Postoperative examination of lymph nodes}

A surgeon examined the lymph nodes around each patient's tumor site immediately after surgery, sorted them by their location (marked beginning at no. 1), and then sent them for a pathological examination. Patients were divided into two groups according to the presence of central lymph node metastasis. "Central lymph node" was defined by Ikoma et al. [8], which included common hepatic artery, celiac artery, and proximal splenic artery LNs (station nos. 8, 9, and 11p). The pathological stage was determined by the 8th edition of TNM staging system of American Joint Commission on Cancer (AJCC) [11].

\section{Statistical methods}

Continuous variables between CnLN-positive and CnLN-negative group were compared using the MannWhitney test, and Fisher's exact test or the chi-square test was used to compare the differences between categorical variables. Potential variables were verified by multivariate analysis using binary logistic regression. Disease-free survival (DFS) was calculated using the Kaplan-Meier method. DFS was defined as the date from curative surgery of the primary tumor to the date of first relapse or death from any cause. Univariate and multivariate Cox proportional hazards regression models were used to determine independent prognostic factors for gastric cancer patients, and the hazard ratio (HR) and its 95\%CI were estimated. All statistical analyses were performed using SPSS 22.0 statistical software. The $P$-value less than 0.05 was considered to be statistically significant.

\section{Results \\ Patient characteristics}

This patient cohort consisted of 337 females (28.6\%) and 841 males $(71.4 \%)$, with average age of 58.54 years (range $7-84)$. A total of 342 patients $(29.0 \%, 342 / 1178)$ had been proven to have $\mathrm{CnLN}$ metastasis, and the incidence of 
central lymph node metastasis was relatively common in gastric cancer patients.

The average number of examined CnLNs was 2.31 (range 1-15). Among the patients with central lymph node metastasis, 258 patients had no. $8 \mathrm{LN}$ metastasis (range 1-9), 100 patients had no. 9 LN metastasis (range 1-9) and 64 patients had no. 11 LN metastasis (range $1-12)$.

\section{Factors associated with CnLNs}

Clinicopathologic characteristics of gastric cancer patients with and without central lymph node metastasis were summarized in the Table1. The results indicated that the CnLNs-positive patients had a larger primary tumor size ( $\leq 4 \mathrm{~cm}$ vs $>4 \mathrm{~cm}, 69.3 \%$ vs $55.1 \%, P<0.001)$, larger number of positive lymph nodes $(P<0.001)$, higher proportion of undifferentiated type $(67.5 \%$ vs $60.5 \%$, $P=0.014)$ and signet ring cell histology $(14.0 \%$ vs $8.5 \%$, $P=0.004)$, more frequent lymphatic vessel invasion (37.4\% vs $18.2 \%, P<0.001)$ and more advanced pathological $\mathrm{T}$ stage $(P<0.001)$ as well as $\mathrm{N}$ stage $(P<0.001)$. However, the distribution of other clinicopathologic factors including age, sex, diagnosis year, the number of retrieved lymph nodes, tumor location and resection type were comparable between CnLN-positive and CnLN-negative patients. In a multivariate analysis, larger tumor size $(P<0.001)$, more frequent lymphatic vessel invasion $(P<0.001)$, signet ring cell histology $(P=0.014)$, and more advanced pathological $\mathrm{T}$ stage $(P=0.013)$ were the significant risk factors for CnLN metastasis (Table 2).

\section{Survival analysis}

The average follow-up time was 39.91 months (range 1 to 178). The DFS rate for all 1178 enrolled patients was $82.5 \%$ at 1 year, $53.3 \%$ at 3 years, and $44.0 \%$ at 5 years. The CnLN-positive patients experienced a poorer survival than CnLN-negative patients (3-year DFS: $30.2 \%$ vs $63 \%$; 5 -year DFS: $18.5 \%$ vs $55.3 \%$; $P<0.001)$. KaplanMeier curves for DFS are illustrated in Fig. 1. Moreover, the survival outcome of the patients with more than three positive CnLNs was worse than that of those with 1 or 2 positive CnLNs (3-year DFS: $19.0 \%$ vs $34.5 \%$; 5 -year DFS: $11.1 \%$ vs $21.4 \%, P=0.001$ ), Kaplan-Meier curves for DFS based on the numbers of positive CnLNs are shown in Fig. 2. In the subgroup analysis, the 5-year DFS rate of $\mathrm{CnLN}$-positive and $\mathrm{CnLN}$-negative patients with N1 stage was $42.3 \%$ and $50.6 \%$, respectively; there was no significant survival difference between two patient $\operatorname{groups}(P=0.376)$. For the N2 stage patients, the 5 -year DFS rate of $\mathrm{CnLN}$-positive and CnLN-negative patients was $25.9 \%$ and $39.3 \%$, respectively $(P=0.017)$; the similar finding was observed in N3 patients (5-year DFS rate, CnLN-positive $11.5 \%$ vs CnLN-negative patients
$23.4 \%, P=0.005)$. Central lymph node metastasis had a significant prognostic significance for N2 and N3 stage patients. Kaplan-Meier curves for DFS based on the $\mathrm{N}$ stage are shown in Fig. 3.

Table 3 showed the results of Cox univariate and multivariate analysis for DFS. According to the univariate analysis, $\mathrm{CnLN}$ metastasis $(P<0.001)$, tumor size $(P<0.001)$, lymphatic vessel invasion $(P<0.001)$, resection type $(P<0.001)$, tumor location $(P<0.001)$, histology type $(P<0.001)$, signet ring cell histology $(P<0.001)$, pathological $\mathrm{T}$ stage $(P<0.001)$ and $\mathrm{N}$ stage $(P<0.001)$, and adjuvant chemotherapy $(P=0.049)$ were found to be significantly associated with poor survival outcome in gastric cancer patients. The multivariate analysis demonstrated that CnLN metastasis (HR:1.366, 95\% CI 1.138-1.640, $P<0.001)$ as well as lymphatic vessel invasion (HR:1.402, 95\% CI 1.168-1.683, $P<0.001$ ), subtotal gastrectomy (HR:0.639, 95\% CI 0.511-0.799, $P<0.001$ ), undifferentiated type (HR 1.249, 95\% CI 1.035-1.507, $P=0.021$ ), signet ring cell histology (HR 1.206, 95\% CI 1.001-1.452, $P=0.048$ ), pathological T stage (T3 stage, HR:1.979, 95\% CI 1.465-2.673, $P<0.001 ;$ T4 stage, HR:2.218, 95\% CI 1.617-3.043, $P<0.001)$ and $N$ stage (N1 stage, HR:2.858, 95\% CI 2.047-3.990, $P<0.001$; N2 stage, HR:3.373, 95\% CI 2.451-4.640, $P<0.001$; N3 stage, HR:5.469, 95\% CI 3.967-7.541, $P<0.001)$, and adjuvant chemotherapy (HR 0.797, 95\% CI 0.663-0.959, $P=0.016$ ) were independent prognostic factors for DFS in gastric cancer patients.

\section{Discussion}

R0 resection with D2 lymphadenectomy is significantly associated with improved survival outcome and widely used as a standard treatment for advanced gastric cancer patients in Eastern countries, especially China and Japan $[9,12,13]$. CnLNs (nos. 8, 9, and 11p) located in the extraperigastric area are included in the extent of D2 lymph node dissection for gastric cancer patients and routinely resected in clinical practice. Through pathologists' hard work, tumors were staged correctly according to the TNM classification. To date, the definition of $\mathrm{N}$ stage was based on the number of positive lymph nodes. The later the $\mathrm{N}$ stage is, the poorer the prognosis. Few studies have focused on the location of positive lymph nodes and its impact on survival outcome in gastric cancer patients $[5,8]$. However, whether the special locations of metastatic lymph nodes was associated with poor survival outcome of the patients treated with D2 lymphadenectomy remain controversial. In the present study, we found that CnLN metastasis was an independent prognostic factor for survival outcome in gastric cancer patients, especially when more than three positive CnLNs were observed $(P=0.001)$. 
Table 1 Background characteristics of the patient, overall and by $\mathrm{CnLN}$ examination status $(\mathrm{N}=1178)$

\begin{tabular}{|c|c|c|c|c|}
\hline \multirow[t]{2}{*}{ Variable } & \multicolumn{3}{|l|}{ CnLN examined } & \multirow[t]{2}{*}{$P$ value } \\
\hline & All patients $(\mathrm{N}=1178)$ & Positive $(\mathrm{N}=342)$ & Negative $(\mathrm{N}=836)$ & \\
\hline Age, average, years & $58.54(7-84)$ & $58.39(23-81)$ & $58.60(7-81)$ & 0.258 \\
\hline$<60, N(\%)$ & $582(49.4 \%)$ & $169(49.4 \%)$ & $413(49.4 \%)$ & 0.997 \\
\hline$\geq 60, \mathrm{~N}(\%)$ & $596(50.6 \%)$ & $173(50.6 \%)$ & $423(50.6 \%)$ & \\
\hline \multicolumn{5}{|l|}{ Gender, N (\%) } \\
\hline Female & $337(28.6 \%)$ & $98(28.7 \%)$ & $239(28.6 \%)$ & 0.517 \\
\hline Male & $841(71.4 \%)$ & $244(71.3 \%)$ & $597(71.4 \%)$ & \\
\hline \multicolumn{5}{|l|}{ Diagnosed year } \\
\hline 2004-2006 & $185(15.7 \%)$ & $61(17.8 \%)$ & $124(14.8 \%)$ & 0.245 \\
\hline 2007-2009 & $359(30.5 \%)$ & $109(31.9 \%)$ & $250(29.9 \%)$ & \\
\hline 2010-2012 & $634(53.8 \%)$ & $172(50.3 \%)$ & $462(55.3 \%)$ & \\
\hline \multicolumn{5}{|l|}{ Tumor size } \\
\hline$\leq 4 \mathrm{~cm}$ & $480(40.7 \%)$ & $105(30.7 \%)$ & $375(44.9 \%)$ & $<0.001$ \\
\hline$>4 \mathrm{~cm}$ & $698(59.3 \%)$ & $237(69.3 \%)$ & $461(55.1 \%)$ & \\
\hline Number of examined LN, average & $22.74(3-119)$ & $25.70(4-119)$ & $21.53(3-82)$ & 0.174 \\
\hline Number of positive $L N$, average & $6.01(1-118)$ & $12.20(1-118)$ & $3.47(3-79)$ & $<0.001$ \\
\hline \multicolumn{5}{|l|}{ Type of resection } \\
\hline Total gastrectomy & $202(17.1 \%)$ & $63(18.4 \%)$ & $139(16.6 \%)$ & 0.254 \\
\hline Subtotal gastrectomy & $976(82.9 \%)$ & $279(81.6 \%)$ & $697(83.4 \%)$ & \\
\hline \multicolumn{5}{|l|}{ Location } \\
\hline Antrum/pylorus & $620(52.6 \%)$ & $183(53.5 \%)$ & $437(52.3 \%)$ & 0.428 \\
\hline Body/fundus & $124(10.5 \%)$ & $32(9.4 \%)$ & $92(11.0 \%)$ & \\
\hline GEJ/cardia & $152(12.9 \%)$ & $38(11.1 \%)$ & $114(13.6 \%)$ & \\
\hline Total & $282(23.9 \%)$ & $89(26.0 \%)$ & $193(23.1 \%)$ & \\
\hline \multicolumn{5}{|l|}{ Histology grade } \\
\hline Differentiated & $441(37.4 \%)$ & $111(32.5 \%)$ & $330(39.5 \%)$ & 0.014 \\
\hline Un-differentiated & $737(62.6 \%)$ & $231(67.5 \%)$ & $506(60.5 \%)$ & \\
\hline \multicolumn{5}{|l|}{ Signet ring cell } \\
\hline Yes & $119(10.1 \%)$ & $48(14.0 \%)$ & $71(8.5 \%)$ & 0.004 \\
\hline No & 1059 (89.9\%) & $294(86.0 \%)$ & 765 (91.5\%) & \\
\hline \multicolumn{5}{|l|}{ Lymphatic vessel invasion } \\
\hline Yes & $280(23.8 \%)$ & $128(37.4 \%)$ & $152(18.2 \%)$ & $<0.001$ \\
\hline No & $898(76.2 \%)$ & $214(62.6 \%)$ & $684(81.8 \%)$ & \\
\hline \multicolumn{5}{|l|}{ Pathological T stage } \\
\hline $\mathrm{T} 2$ & $220(18.7 \%)$ & $41(12.0 \%)$ & $179(21.4 \%)$ & $<0.001$ \\
\hline T3 & $666(56.5 \%)$ & $194(56.7 \%)$ & $472(56.5 \%)$ & \\
\hline $\mathrm{T} 4$ & $292(24.8 \%)$ & $107(31.3 \%)$ & $185(22.1 \%)$ & \\
\hline \multicolumn{5}{|l|}{ Pathological N stage } \\
\hline NO & $323(27.4 \%)$ & $0(0.0 \%)$ & $323(38.6 \%)$ & $<0.001$ \\
\hline N1 & $219(18.6 \%)$ & 35 (10.2\%) & 184 (22.0\%) & \\
\hline N2 & $265(22.5 \%)$ & 84 (24.6\%) & $181(21.7 \%)$ & \\
\hline N3 & 371 (31.5\%) & $223(65.2 \%)$ & 148 (17.7\%) & \\
\hline
\end{tabular}

Ikoma et al. defined "central lymph node" as common hepatic artery, celiac artery, and proximal splenic artery LNs (station nos. 8, 9, and 11p) [8]. In our study, CnLN metastasis was relatively common (29.0\%) (21.9\% in no. 8 LNs, $8.4 \%$ in no. 9 LNs, and $5.4 \%$ in no. 11p LNs) and was significantly associated with poor survival outcome. And in this trial, larger tumor size $(P<0.001)$, more frequent lymphatic vessel invasion $(P<0.001)$, signet ring cell histology $(P=0.014)$, and more advanced pathological $\mathrm{T}$ stage $(P=0.013)$ were 
Table 2 Multivariate logistic analysis for CnLN metastasis

\begin{tabular}{lllr}
\hline Variable & Odds ratio & 95\%Cl & $P$ value \\
\hline Gender (female vs male) & 1.077 & $0.800-1.449$ & 0.625 \\
Age $\geq 60$ year vs $<60$ year) & 1.026 & $0.786-1.340$ & 0.850 \\
Tumor size ( 4 cm vs $\leq 4$ cm) & 1.689 & $1.266-2.254$ & $<0.001$ \\
Lymphatic vessel invasion (yes vs no) & 2.558 & $1.911-3.425$ & $<0.001$ \\
Type of resection (total vs subtotal gastrectomy) & 1.117 & $0.748-1.668$ & \\
Location (ref. antrum/pylorus) & & & 0.589 \\
Body/fundus & 0.806 & $0.494-1.317$ \\
GEJ/cardia & 0.649 & $0.421-1.000$ \\
Total & 0.822 & $0.572-1.181$ \\
Histology type (un-differentiated vs differentiated) & 1.093 & $0.815-1.466$ \\
Signet ring cell (yes vs no) & 1.708 & $1.117-2.611$ \\
Pathological T stage (ref. T2) & & & 0.390 \\
T3 & 1.565 & $1.051-2.330$ \\
T4 & 1.949 & $1.248-3.036$ \\
\hline
\end{tabular}
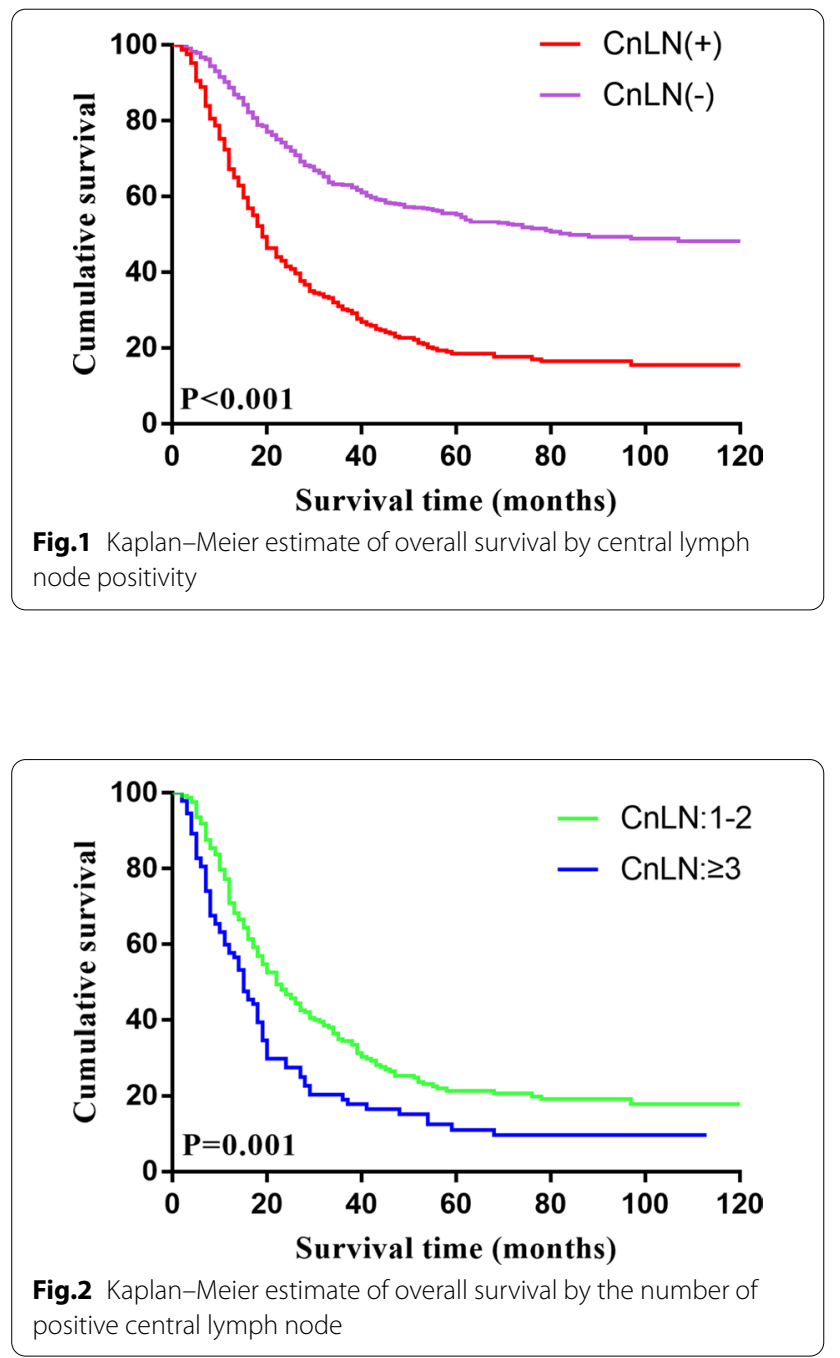

significantly related to CnLNs metastasis. In the subgroup analysis, we found that CnLN metastasis was associated with shorter 5-year DFS in $\mathrm{pN} 2$ and $\mathrm{pN} 3$ patients (for $\mathrm{pN} 2$ patients: $25.9 \%$ vs $39.3 \%, P=0.017$; for pN3: $11.5 \%$ vs $23.4 \%, P=0.005$ ), but not showed a significant difference for $\mathrm{pN} 1$ patients $(42.3 \%$ vs $50.6 \%$, $P=0.326)$. Central lymph node metastasis is predictive of prognosis for $\mathrm{pN} 2 / 3$ patients.

For pN1 patients, CnLN metastasis was not significantly associated with survival outcome, which might be related to the low metastatic rate and the mechanism of "skip metastasis" [14, 15]. Skip metastasis was defined when LN metastasis appeared to bypass or skip tiers rather than following the lymphatic streams and was not related to the location of the primary tumor. In earlier tumor stages, tumor cell colonization might be random, and studies have shown that station nos. $1,7,8 \mathrm{a}, 9$, and 11 were the main sites of skip metastasis [16]. The pN1 stage patients with positive CnLNs were likely to experience skip metastasis. However, the association between skip metastasis and survival outcome in gastric cancer patients remains a matter of debate. Some studies have revealed that skip metastasis has no impact on survival $[14,16]$. And our study supported this point, because CnLN metastasis in $\mathrm{pN} 1$ stage patients was not related to survival $(P=0.376)$. Skip metastasis might be the reason for which CnLNpositive $\mathrm{pN} 1$ patients did not experience poor survival rates, similar to $\mathrm{pN} 2 / 3$ patients.

According to the 5th Japanese gastric cancer treatment guideline, the National Comprehensive Cancer Network (NCCN), and the current American Joint 


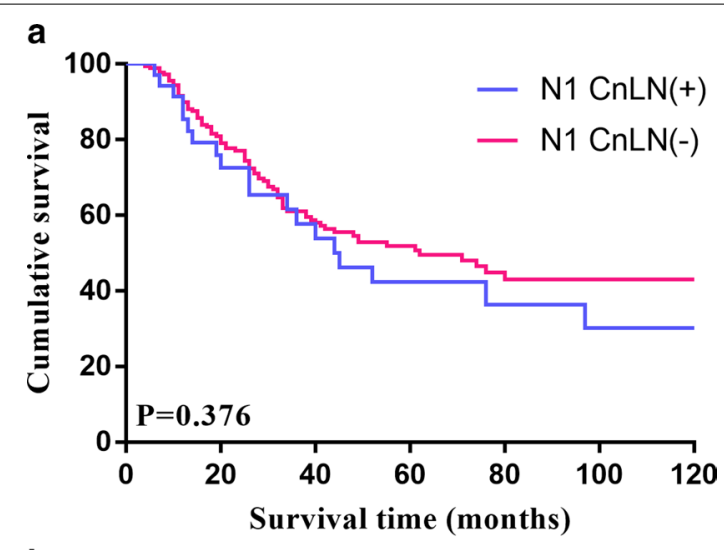

b

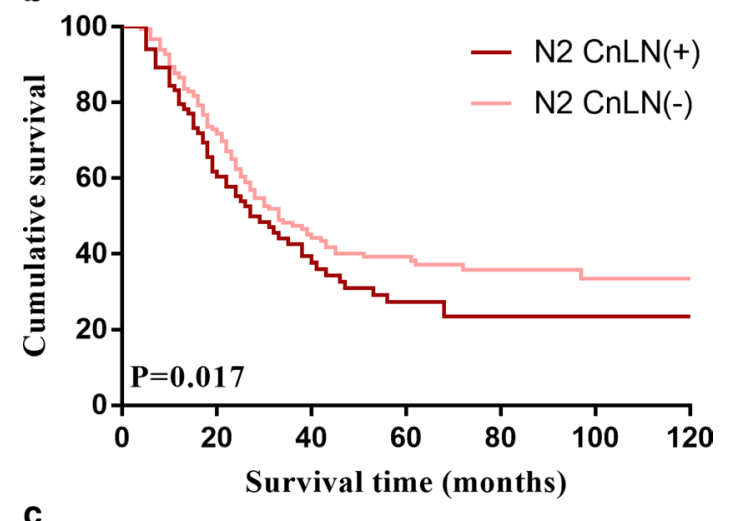

C

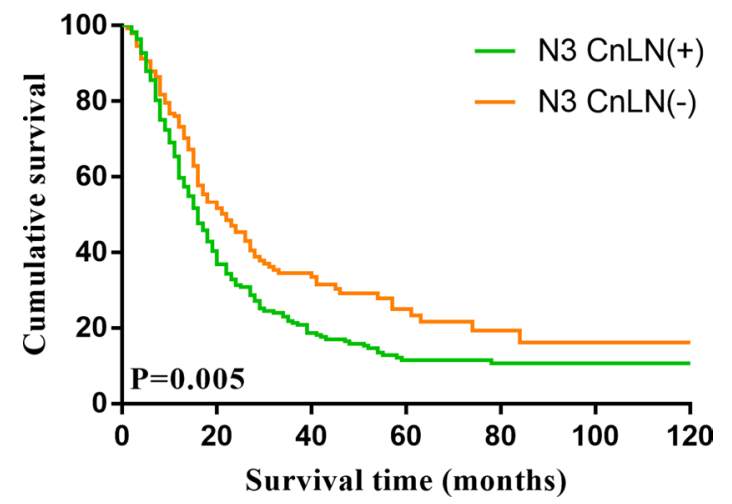

Fig.3 Kaplan-Meier estimate of overall survival by pathological $N$ stage and central lymph node positivity

Committee on Cancer (AJCC) staging system (8th edition), $\mathrm{N}$ stage is defined by the number, rather than the region, of positive lymph nodes among the regional lymph nodes, which is a consistent and effective method used worldwide [4, 11]. However, for patients with positive lymph nodes, the location of the metastatic LNs, especially central lymph nodes, is strongly correlated with survival $[17,18]$. And in our study, we found the GC patients who located in the same $\mathrm{N}$ stage showed the different survival outcome because of CnLN metastasis. Especially for pN2 and pN3 stage, patients with positive CnLNs had shorter lifetime significantly. As a result, the $\mathrm{N}$ stage as well as central lymph nodes metastasis, both accommodate substantial importance when exploring the comprehensive treatment and predicting the prognosis for advanced gastric cancer patients. Additionally, central lymph nodes metastasis could serve as a potential supplement to the current international $\mathrm{N}$ stage for evaluating the prognosis of GC patients more accurately.

CnLN metastasis could be a potential predictor for prognosis and help guide postoperative treatment. Therefore, D2 lymphadenectomy and an accurate LN pathological examination are necessary for advanced gastric cancer patients [19]. Extensive lymphadenectomy could resect the micrometastasis and decrease the recurrence rate, especially for upper gastric cancer. Some studies have reported that upper gastric carcinoma is more prone to LN metastasis, especially at station nos. 1, 2, 3, and 7 , and usually metastasizes to the para-aortic lymph node through the left gastric cancer artery and splenic artery $[20,21]$. Thus, surgeons should carefully examine the lymph node and decide whether they need to perform a more extensive lymphadenectomy. In contrast to Eastern countries, D1 lymphadenectomy is more common in Western countries, mainly because of its lower rates of poorly differentiated histology and proximal stomach involvement-factors that are related to poor survival [6]. The standard range of lymphadenectomy is still under debate, but we suggest that patients with a later stage (i.e., later than T2) and with suspicious positive LNs should undergo extensive lymphadenectomy.

In clinical practice, gastric cancer patients, particularly those whose postoperative pathology reports have showed positive lymph nodes, tumor invaded the serosa and later TNM stage, are recommended to undergo adjuvant chemotherapy due to higher recurrent rate and poorer prognosis. Extensive and highquality dissection and an accurate lymph node stage are key factors to consider when planning postoperative treatment. Guidelines request at least 15 lymph nodes to be examined in D2 resection for accurate LN staging [22]. And the later N stage, the more possible to metastasize to extraperigastric area following the lymphatic streams. In our study, we found that patients with more than three positive CnLNs had a poorer prognosis than the patients with one or two metastatic CnLNs $(P=0.001)$. Thus, in D2-resected GC patients in N2 stage or later, with positive CnLNs, especially at least three-all the variables related to a heavy lymph burden,-adjuvant chemoradiotherapy should initiate timely after surgery to eradicate micrometastasis and prolong survival. Some phase III studies have revealed 
Table 3 Univariate and multivariate Cox analysis of disease-free survival (DFS) in 1178 D2-resected gastric cancer patients

\begin{tabular}{|c|c|c|c|c|}
\hline \multirow[t]{2}{*}{ Variable } & \multicolumn{2}{|l|}{ Univariate analysis } & \multicolumn{2}{|l|}{ Multivariate analysis } \\
\hline & $\mathrm{HR}(95 \% \mathrm{Cl})$ & $P$ value & HR $(95 \% C l)$ & $P$ value \\
\hline CnLN metastasis (positive vs negative ) & $2.697(2.290-3.176)$ & $<0.001$ & $1.366(1.138-1.640)$ & 0.001 \\
\hline Age ( $\geq 60$ years vs $<60$ years) & $1.036(0.882-1.217)$ & 0.149 & - & - \\
\hline LN examined $(\geq 15$ vs $<15)$ & $1.246(0.970-1.599)$ & 0.085 & - & - \\
\hline Tumor size (> 4 cm vs $\leq 4 \mathrm{~cm})$ & $1.592(1.342-1.890)$ & $<0.001$ & $0.991(0.823-1.192)$ & 0.922 \\
\hline Lymphatic vessel invasion (yes vs no) & $2.295(1.930-2.730)$ & $<0.001$ & $1.402(1.168-1.683)$ & $<0.001$ \\
\hline Type of resection (total vs subtotal gastrectomy) & $0.507(0.419-0.613)$ & $<0.001$ & $0.639(0.511-0.799)$ & $<0.001$ \\
\hline \multicolumn{5}{|l|}{ Location (ref. antrum/pylorus ) } \\
\hline Body/fundus & $1.056(0.801-1.393)$ & 0.698 & $0.901(0.668-1.215)$ & 0.494 \\
\hline GEJ/cardia & $1.381(1.082-1.762)$ & 0.009 & $1.353(1.052-1.740)$ & 0.019 \\
\hline Total & $1.395(1.151-1.692)$ & 0.001 & $1.024(0.819-1.279)$ & 0.837 \\
\hline Histology type (un-differentiated vs differentiated ) & $1.499(1.258-1.786)$ & $<0.001$ & $1.206(1.001-1.452)$ & 0.048 \\
\hline Signet ring cell (yes vs no) & $1.477(1.170-1.865)$ & $<0.001$ & $1.233(0.962-1.580)$ & 0.098 \\
\hline \multicolumn{5}{|l|}{ Pathological T stage (ref. T2) } \\
\hline T3 & $2.647(1.975-3.547)$ & $<0.001$ & $1.979(1.465-2.673)$ & $<0.001$ \\
\hline T4 & $3.914(2.888-5.304)$ & $<0.001$ & $2.218(1.617-3.043)$ & $<0.001$ \\
\hline \multicolumn{5}{|l|}{ Pathological N stage (ref. N0) } \\
\hline N1 & $3.066(2.207-4.259)$ & $<0.001$ & $2.858(2.047-3.990)$ & $<0.001$ \\
\hline N2 & $4.512(3.320-6.132)$ & $<0.001$ & $3.373(2.451-4.640)$ & $<0.001$ \\
\hline N3 & $8.403(6.296-11.214)$ & $<0.001$ & $5.469(3.967-7.541)$ & $<0.001$ \\
\hline Adjuvant chemotherapy (yes vs no) & $0.834(0.696-0.999)$ & 0.049 & $0.797(0.663-0.959)$ & 0.016 \\
\hline
\end{tabular}

that both adjuvant chemotherapy and radiochemotherapy were beneficial in preventing recurrence in D2-resected GC patients with positive LNs [23, 24]. The oxaliplatin combined with capecitabine (XELOX regimen) is commonly used as first-line postoperative adjuvant chemotherapy, especially for LN-positive gastric cancer patients, with tolerable side effects (most AEs are grade I/II) and a survival benefit (25.4-29 month) [25-27].

There were some limitations in our study. First, our study was a retrospective analysis involving a single institution. In the future, well-designed, large sample size and multicenter studies still need to be performed. Additionally, not all patients had at least 15 examined lymph nodes, which may have caused the incurrent $\mathrm{N}$ stage. Secondly, therapeutic protocols and recommendations for gastric cancer patients could have evolved during the study period. Adjuvant chemotherapy had been successfully performed in the recent decade, but the proportion of the patients who received adjuvant chemotherapy was relatively low in the current cohort. This may have a potential impact on prognostic assessment of gastric cancer patients.

\section{Conclusion}

In conclusion, this study reported that $\mathrm{CnLN}$ metastasis could be regarded as a predictor for survival outcome in gastric cancer patients who underwent R0 resection and D2 lymphadenectomy. The anatomical location of positive LNs may be supplement to "N stage" for accurately prognostic evaluation. Large sample, multicenter, randomized clinical trials are still needed in the future.

\footnotetext{
Abbreviations

GC: Gastric cancer; DFS: Disease-free survival; OS: Overall survival; HR: Hazard ratio; LN: Lymph node; CnLN: Central lymph node; AJCC: American Joint Committee on Cancer; UICC: Union International Against Cancer.
}

\section{Acknowledgements}

Not applicable

\section{Authors' contributions}

Conception and design of study: HL, BZ, BH. Acquisition of data: HL, BZ. Analysis and/or interpretation of data: $\mathrm{HL}, \mathrm{RH}, \mathrm{ZZ}$. Drafting the manuscript: $\mathrm{HL}$, YS, HX. Revising the manuscript critically for important intellectual content: $\mathrm{BH}, \mathrm{BZ}, \mathrm{HX}$. All authors approved the final version of the manuscript to be published and agree to be accountable for all aspects of the work. All authors read and approved the final manuscript. 


\section{Funding}

This work was supported by the Natural Science Foundation of Liaoning province (No. 20180530026).

\section{Availability of data and materials}

The datasets used and/or analysed during the current study are available from the corresponding author on reasonable request.

\section{Ethical approval and informed consents}

No ethical approval or informed consent was required for this study.

\section{Consent for publication}

Not applicable.

\section{Competing interests}

The authors declare that they have no conflict of interest.

\section{Author details}

1 Department of Surgical Oncology, First Affiliated Hospital of China Medical University, No. 155 Nanjing North Street, Heping District, Shenyang 110001, People's Republic of China. ${ }^{2}$ Department of Clinical Medicine of Year 2017, Dalian Medical University, Dalian, People's Republic of China.

Received: 21 September 2020 Accepted: 9 December 2020 Published online: 06 January 2021

\section{References}

1. Bray F, Ferlay J, Soerjomataram I, Siegel RL, Torre LA, Jemal A. Global cancer statistics 2018: GLOBOCAN estimates of incidence and mortality worldwide for 36 cancers in 185 countries. CA A Cancer JClin. 2018;68(6):394-424.

2. Rohde H, Gebbensleben B, Bauer P, Stutzer H, Zieschang J. Has there been any improvement in the staging of gastric cancer? Findings from the German Gastric Cancer TNM Study Group. Cancer. 1989;64(12):2465-81.

3. Japanese Gastric Cancer Association. Japanese classification of gastric carcinoma: 3rd English edition. Gastric Cancer. 2011;14(2):101-12.

4. Hayashi H, Ochiai T, Suzuki T, et al. Superiority of a new UICC-TNM staging system for gastric carcinoma. Surgery. 2000;127(2):129-35.

5. Hidaka H, Eto T, Maehara N, Jimi S, Hotokezaka M, Chijiiwa K. Comparative effect of lymph node metastasis classified by the anatomical site or by the number of nodes involved on prognosis of patients with gastric cancer. Hepatogastroenterology. 2008;55(88):2269-72.

6. Karpeh MS, Leon L, Klimstra D, Brennan MF. Lymph node staging in gastric cancer: is location more important than number? An analysis of 1,038 patients. Ann Surg. 2000;232(3):362-71.

7. Katai H, Yoshimura K, Maruyama K, Sasako M, Sano T. Evaluation of the new international union against cancer TNM staging for gastric carcinoma. Cancer. 2000;88(8):1796-800.

8. Ikoma N, Estrella JS, Blum M, et al. Central lymph node metastasis in gastric cancer is predictive of survival after preoperative therapy. J Gastrointest Surg. 2018;22(8):1325-33.

9. Songun I, Putter H, Kranenbarg EM, Sasako M, van de Velde CJ. Surgical treatment of gastric cancer: 15-year follow-up results of the randomised nationwide Dutch D1D2 trial. Lancet Oncol. 2010;11(5):439-49.

10. Ikoma N, Blum M, Estrella JS, et al. Left gastric artery lymph nodes should be included in D1 lymph node dissection in gastric cancer. J Gastrointest Surg. 2017;21(10):1563-70.

11. Amin MB, Greene FL, Edge SB, et al. The eighth edition AJCC cancer staging manual: continuing to build a bridge from a population-based to a more "personalized" approach to cancer staging. CA Cancer J Clin. 2017;67(2):93-9.

12. Mocellin S, Nitti D. Lymphadenectomy extent and survival of patients with gastric carcinoma: a systematic review and meta-analysis of time-toevent data from randomized trials. Cancer Treat Rev. 2015;41(5):448-54

13. de Steur WO, Hartgrink HH, Dikken JL, Putter $H$, van de Velde CJ. Quality control of lymph node dissection in the Dutch Gastric Cancer Trial. Br J Surg. 2015;102(11):1388-93.

14. Liu JY, Deng JY, Zhang NN, et al. Clinical significance of skip lymph-node metastasis in pN1 gastric-cancer patients after curative surgery. Gastroenterol Rep. 2019;7(3):193-8.

15. Choi YY, An JY, Guner A, et al. Skip lymph node metastasis in gastric cancer: is it skipping or skipped? Gastric Cancer. 2016;19(1):206-15.

16. Park SS, Ryu JS, Min BW, et al. Impact of skip metastasis in gastric cancer. ANZ J Surg. 2005;75(8):645-9.

17. Son T, Hyung WJ, Kim JW, et al. Anatomic extent of metastatic lymph nodes: still important for gastric cancer prognosis. Ann Surg Oncol. 2014;21(3):899-907.

18. Chen J, Chen C, He Y, Wu K, Wu H, Cai S. A new pN staging system based on both the number and anatomic location of metastatic lymph nodes in gastric cancer. J Gastrointest Surg. 2014;18(12):2080-8.

19. Griniatsos J, Yiannakopoulou E, Gakiopoulou H, et al. Clinical implications of the histologically and immunohistochemically detected solitary lymph node metastases in gastric cancer. Scand J Surg. 2011;100(3):174-80.

20. Yamashita H, Seto Y, Sano T, Makuuchi H, Ando N, Sasako M. Results of a nation-wide retrospective study of lymphadenectomy for esophagogastric junction carcinoma. Gastric Cancer. 2017;20(Suppl 1):69-83.

21. Cense HA, Sloof GW, Klaase JM, et al. Lymphatic drainage routes of the gastric cardia visualized by lymphoscintigraphy. J Nucl Med. 2004;45(2):247-52.

22. Ajani JA, D'Amico TA, Almhanna K, et al. Gastric cancer, version 3.2016, NCCN clinical practice guidelines in oncology. J Natl Compr Cancer Netw. 2016;14(10):1286-312.

23. Tsuburaya A, Mizusawa J, Tanaka Y, Fukushima N, Nashimoto A, Sasako M. Neoadjuvant chemotherapy with S-1 and cisplatin followed by D2 gastrectomy with para-aortic lymph node dissection for gastric cancer with extensive lymph node metastasis. Br J Surg. 2014;101(6):653-60.

24. Lee J, Lim DH, Kim S, et al. Phase III trial comparing capecitabine plus cisplatin versus capecitabine plus cisplatin with concurrent capecitabine radiotherapy in completely resected gastric cancer with D2 lymph node dissection: the ARTIST trial. J Clin Oncol. 2012;30(3):268-73.

25. Wang Y, Yu YY, Li W, et al. A phase II trial of Xeloda and oxaliplatin (XELOX) neo-adjuvant chemotherapy followed by surgery for advanced gastric cancer patients with para-aortic lymph node metastasis. Cancer Chemother Pharmacol. 2014;73(6):1155-61.

26. Xu HB, Huang F, Su R, Shen FM, Lv QZ. Capecitabine plus oxaliplatin (XELOX) compared with 5-fluorouracil/leucovorin plus oxaliplatin (FOLFOXs) in advanced gastric cancer: meta-analysis of randomized controlled trials. Eur J Clin Pharmacol. 2015;71(5):589-601.

27. Wang Y, Zhuang RY, Yu YY, et al. Efficacy of preoperative chemotherapy regimens in patients with initially unresectable locally advanced gastric adenocarcinoma: capecitabine and oxaliplatin (XELOX) or with epirubicin (EOX). Oncotarget. 2016;7(46):76298-307.

\section{Publisher's Note}

Springer Nature remains neutral with regard to jurisdictional claims in published maps and institutional affiliations. 